\title{
HSulf-1 suppresses cell growth and down-regulates Hedgehog signaling in human gastric cancer cells
}

\author{
HUI-YAN MA ${ }^{1}$, FANG ZHANG ${ }^{2}$, JIE $^{3}{ }^{3}$, MIN-LI MO ${ }^{3}$, ZHAO CHEN $^{3}$, \\ LILI LIU ${ }^{1}$, HAI-MENG ZHOU ${ }^{2,3}$ and QING SHENG ${ }^{1,3}$ \\ ${ }^{1}$ College of Life Sciences, Zhejiang Sci-Tech University, Hangzhou 310018; ${ }^{2}$ Zhejiang Provincial Key Laboratory \\ of Applied Enzymology, Yangtze Delta Region, Institute of Tsinghua University, Jiaxing, \\ Zhejiang 314006; ${ }^{3}$ School of Life Sciences, Tsinghua University, Beijing 100084, P.R. China
}

Received March 15, 2011; Accepted August 12, 2011

DOI: $10.3892 / \mathrm{ol} .2011 .407$

\begin{abstract}
Gastric cancer is the second most lethal cancer worldwide. Despite the current surgical and adjuvant therapies, 5-year survival remains less than $20-25 \%$ in the US, Europe and China. Therefore, there is an urgent need to identify new therapeutic targets for treating this malignant disease. Accumulating evidence has supported that aberrant activation of the Hedgehog signaling pathway plays a crucial role in tumorigenesis and progression of gastric cancer. Human sulfatase-1 (HSulf-1) is a recently identified enzyme that desulfates cell surface heparan sulfate proteoglycans (HSPGs), which is critical for Hedgehog signal transduction under a highly sulfated state. HSulf-1 has recently emerged as a tumor suppressor gene in certain types of cancer, including ovarian, breast, myeloma and hepatocellular carcinoma; however, its role in gastric cancer remains to be elucidated. Therefore, we established HSulf-1-expressing monoclonal MKN28 gastric cancer cells to investigate its function in gastric cancer. Expression of HSulf-1 significantly suppressed cellular proliferation and growth in MKN28 gastric cancer cells. Notably, HSulf-1 inhibits Gli-mediated transcription and down-regulates the expression of Hedgehog target genes, including GLI1, PTCH1/2, HHIP, CCND1, C-MYC and BCL-2. Collectively, the study provides evidence that HSulf-1 may function as a tumor suppressor in gastric cancer. It suppresses gastric cancer cell proliferation, possibly through abrogating the Hedgehog signaling pathway. The study provides new mechanistic insight into HSulf-1mediated tumor suppression, and supports the use of HSulf-1 as a potential new therapeutic target in treating gastric cancer.
\end{abstract}

Correspondence to: Professor Qing Sheng, College of Life Sciences, Zhejiang Sci-Tech University, Hangzhou 310018, P.R. China E-mail: csheng@zstu.edu.cn

Key words: HSulf-1, Hedgehog signaling, gastric cancer, proliferation

\section{Introduction}

Gastric cancer is the second most malignant type of cancer worldwide. It is often diagnosed at a late stage, and despite the current regimen of surgical, chemo-, radio- and other adjuvant therapies, gastric cancer has a poor prognosis with a 5-year survival rate of less than $20-25 \%$ in the USA, Europe and China (1). This has galvanized efforts to identify new therapeutic targets for treating this lethal disease.

Emerging evidence revealed that gastric cancer results from a combination of factors, including Helicobacter pylori-induced inflammation and deregulation of the Wnt and Hedgehog signaling pathways (2-4). The Hedgehog (Hh) family of proteins controls multiple cellular functions, including cell growth, survival and outcome, as well as body patterning and organ morphogenesis (5). Deregulated Hh pathway activation plays a role in various types of cancer including glioma, basal cell carcinoma, medulloblastoma, lung, breast, pancreatic and gastric cancers (6-8). Hh signaling is controlled by two transmembrane proteins, Patched (Ptch1) and Smoothened (SMO). In the absence of the Hh ligand, PTCH1 inhibits SMO, causing cleavage of GLI1 to the N-terminal repressor form. Once Hh binds to PTCH1, the inhibitory effect on SMO is released, causing active full-length GLI1 to transport into the nucleus and activate transcription of the $\mathrm{Hh}$ target genes, including GLI1, PTCH1, HHIP and C-MYC (9-11).

Human sulfatase-1 (HSulf-1) has emerged as a negative regulator of $\mathrm{Hh}$ signaling. It disrupts the distribution of the Hh morphogen and Hh signaling transduction by desulfating cell surface heparan sulfate proteoglycans (HSPGs) (12-14). HSulf-1 was cloned as the first human ortholog of the developmentally regulated putative Quail sulfatase QSulf-1 (13) and was found to be downregulated in a number of cancer cells including ovarian, breast, renal tumor, hepatocellular carcinoma, myeloma and head and neck squamous carcinoma (15-21). HSulf-1 expression inhibited cancer cell growth, cell motility and invasion in multiple cancer cells, and promoted stress-induced apoptosis (15-17). Furthermore, HSulf-1 expression led to a reduction in vascular density and increased breast cancer cell apoptosis in xenografts, suggesting that HSulf-1 inhibits both angiogenesis and tumorigenesis 
Table I. Primers for semi-quantitative RT-PCR analysis.

Gene name

Primer sequences (5'-3')

Amplicon size (bp)

\begin{tabular}{|c|c|c|}
\hline GLI1 & $\begin{array}{l}\text { 5'-TACTCACGCCTCGAAAACCT-3' (forward) } \\
\text { 5'-GTCTGCTTTCCTCCCTGATG-3' (reverse) }\end{array}$ & 352 \\
\hline PTCH1 & $\begin{array}{l}\text { 5'-ATGCTGGCGGGATCTGAGTTCGACT-3' (forward) } \\
\text { 5'- GGGTGTGGGCAGGCGGTTCAAG-3' (reverse) }\end{array}$ & 174 \\
\hline PTCH2 & $\begin{array}{l}\text { 5'- GATGTGCTCTGCTGCTTCTCCA-3' (forward) } \\
\text { 5'- CTGCCTTCTGCCTTGTCTCCTC-3' (reverse) }\end{array}$ & 283 \\
\hline HHIP & $\begin{array}{l}\text { 5'-GCCTCGCATTCCATCCCAATTAC-3' (forward) } \\
\text { 5'-ACCCATCCATTTCTTCCATATCATCC-3' (reverse) }\end{array}$ & 297 \\
\hline C-MYC & $\begin{array}{l}\text { 5'-TTCGGGTAGTGGAAAACCAG-3' (forward) } \\
\text { 5'-CAGCAGCTCGAATTTCTTCC-3' (reverse) }\end{array}$ & 203 \\
\hline MYCN & $\begin{array}{l}\text { 5'-CATCCACCAGCAGCACAACTAT-3' (forward) } \\
\text { 5'-CTCAAGCTCTTAGCCTTTGGGG-3' (reverse) }\end{array}$ & 138 \\
\hline CCND1 & $\begin{array}{l}\text { 5'-AGCTCCTGTGCTGCGAAGTGGAAAC-3' (forward) } \\
\text { 5'-AGTGTTCAATGAAATCGTGCGGGGT-3' (reverse) }\end{array}$ & 480 \\
\hline FOXF1 & $\begin{array}{l}\text { 5'-GCCGTATCTGCACCAGAACA-3' (forward) } \\
\text { 5'-CGTTGAAAGAGAAGACAAACTCCTT-3' (reverse) }\end{array}$ & 116 \\
\hline FOXM1 & $\begin{array}{l}\text { 5'-GGAGCAGCGACAGGTTAAGG-3' (forward) } \\
\text { 5'-GTCGTGCAGGGAAAGGTTGT-3' (reverse) }\end{array}$ & 244 \\
\hline BCL2 & $\begin{array}{l}\text { 5'-TTTGAGTTCGGTGGGGTCAT-3' (forward) } \\
\text { 5'-TGACTTCACTTGTGGCCCAG-3' (reverse) }\end{array}$ & 275 \\
\hline 18S rRNA & $\begin{array}{l}\text { 5'-CAGCCACCCGAGATTGAGCA-3' (forward) } \\
\text { 5'-TAGTAGCGACGGGCGGTGTG-3' (reverse) }\end{array}$ & 254 \\
\hline
\end{tabular}

in vivo $(18,19)$. However, the role of HSulf-1 in gastric cancer tumorigenesis remains to be elucidated. Findings of our previous study demonstrated that the HSulf-1 expression is downregulated in gastric cancer cells, and that HSulf-1 gene silencing is associated with a high level of promoter hypermethylation (22).

In this study, we also investigated the function of HSulf-1 in gastric cancer cell proliferation. Expression of HSulf-1 in monoclonal MKN28 gastric cancer cells suppressed cell proliferation and colony formation. Notably, HSulf-1 inhibited GLI1-mediated transcription and the downregulated expression of Hh downstream target genes, including GLI1, PTCH1, C-MYC, CCND1 and BCL2. These data strongly support that HSulf-1 may inhibit gastric cancer cell proliferation by blocking the Hh pathway.

\section{Materials and methods}

Cell culture and establishment of HSulf-expressing monoclonal MKN28 stable cells. The human gastric cancer cell line MKN28 was obtained from the China Center for Type Culture Collection (Wuhan, China). Cells were cultured in Dulbecco's modified Eagle's medium (DMEM) supplemented with $10 \%$ fetal bovine serum (FBS), penicillin (100 IU/ml) and streptomycin $(100 \mu \mathrm{g} / \mathrm{ml})$ in a humidified incubator at $37^{\circ} \mathrm{C}$ with a $5 \% \mathrm{CO}_{2}$ atmosphere. Cells $\left(1 \times 10^{4}\right)$ were plated in 12 -well plates one day prior to transfection. Monoclonal cells stably expressing HSulf-1 or empty vector were established following plasmid transfection and Geneticin (G418 sulfate) selection.
The stable monoclonal cell lines were then cultured in DMEM supplemented with $10 \% \mathrm{FBS}$ and $0.5 \mathrm{mg} / \mathrm{ml} \mathrm{G} 418$. Cells were maintained at $37^{\circ} \mathrm{C}$ in a humid incubator with $5 \% \mathrm{CO}_{2}$.

Quantitative real-time PCR (RT-PCR). RNA extraction and RT-PCR were performed as previously described (22). The primer sequences were as follows: 18S rRNA, 5'-CAGCCA CCCGAGATTGAGCA-3' (forward) and 5'-TAGTAGCGACG GGCGGTGTG-3' (reverse); HSulf-1, 5'-ACTGTACCAATCG GCCAGAG-3' (forward) and 5'-CCTCCTTGAATGGGTGA AGA-3' (reverse). Semi-quantitative RT-PCR was used to examine the expression levels of Hh downstream target genes. The primers used are shown in Table I. The procedure was performed as described in a previous study (22).

Immunoblotting. For total cell lysates, the cell pellet was resuspended in RIPA buffer (50 mM Tris $\mathrm{pH} 8.0,150 \mathrm{mM}$ $\mathrm{NaCl}, 1 \%$ Triton $\mathrm{X}-100,0.5 \%$ sodium deoxycholate and $0.1 \%$ SDS), mixed with protease inhibitors and vortexed for $5 \mathrm{sec}$. Following incubation on ice for $15 \mathrm{~min}$, the suspension was centrifuged at $14,000 \mathrm{rpm}$ at $4^{\circ} \mathrm{C}$ for $5 \mathrm{~min}$ and the supernatant was collected for immunoblotting. Total cell lysates $(20 \mu \mathrm{g})$ were subjected to SDS-PAGE and transferred to a PVDF membrane (GE Healthcare, Chalfont, UK). The membrane was blocked by $5 \%$ milk for $2 \mathrm{~h}$ and incubated with primary antibodies overnight at $4{ }^{\circ} \mathrm{C}$, followed by horseradish peroxidase-conjugated secondary antibody (Pierce, Rockford, IL, USA) and Super Signal West Pico reagent (Pierce). The primary antibodies included anti-HSulf-1 (1:250; Abnova 
A

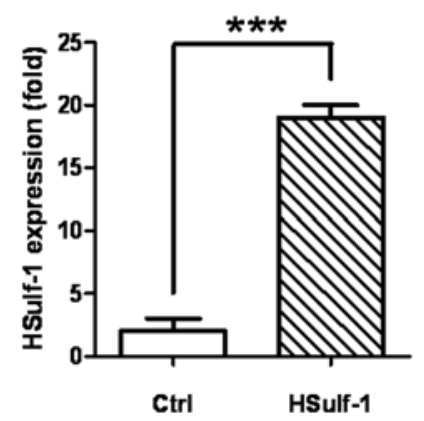

B

\section{Ctrl HSulf-1}

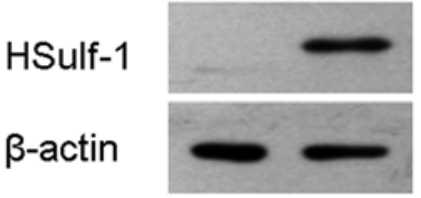

Figure 1. Expression of HSulf-1 in stable-transfected MKN28 cells. (A) Quantitative RT-PCR showing the expression of HSulf-1 mRNA in MKN28-HSulf-1 stable cells (HSulf-1) and the empty vector control cells (Ctrl). (B) Immunoblotting showing the HSulf-1 protein expression in MKN28-HSulf-1 cells (lane 2) vs. the empty vector control (lane 1). ${ }^{* * *} \mathrm{p}<0.001$ (Student's t-test). Error bars, \pm SD. Data are representative of at least three independent experiments with similar results.

Corp., Walnut, CA, USA) and anti- $\beta$-actin (1:5000; Sigma Aldrich, St. Louis, MO, USA).

Cell proliferation assay. Cell growth rates were determined by a CellTiter $96^{\circledR}$ AQueous non-radioactive cell proliferation assay kit (Promega, Madison, WI, USA). Briefly, the stable cell lines were plated into 96-well tissue culture plates at $5 \times 10^{2}$ cells/well. After the cells were cultured for a number of days as indicated, MTS solutions were added to the medium and incubated for $1.5 \mathrm{~h}$ at $37^{\circ} \mathrm{C}$. The absorbance at $490 \mathrm{~nm}$ was measured using a microplate reader model 680 (Bio-Rad, Hercules, CA, USA) and the normalized absorbance was plotted against culture time to determine the number of viable cells.

Colony formation assay. The stable cells expressing empty vector or HSulf-1 were seeded in triplicate in 100-mm dishes at $1 \times 10^{3}$ cells/dish. Fresh medium containing $0.5 \mathrm{mg} / \mathrm{ml}$ G418 was changed every 3-4 days. After being cultured for 2-3 weeks, cells were fixed with $4 \%$ paraformaldehyde for 10 min, washed with PBS several times, stained with $0.1 \%$ crystal violet for $20 \mathrm{~min}$ and photographed. Clones with more than 50 cells were counted.

Luciferase reporter assay. MKN28 cells at a density of $5 \times 10^{3}$ cells/well were seeded into 24 -well plates prior to transfection. 8xGli-BS or 8xmutGli-BS luciferase reporter plasmid was co-transfected with $20 \mathrm{ng}$ TK-Renilla (pRL-TK) (Promega) internal control plasmid and DNA expression vectors as indicated. After cells were cultured for $48 \mathrm{~h}$, the luciferase activity was measured by the Dual-Glo Luciferase Assay System (Promega). The ratio between firefly luciferase activity (8xGli or Mut Gli) and renilla activity was calculated to assess the Gli transcriptional activity.

Statistical analysis. Data are expressed as the standard error of the mean (SEM) unless specifically indicated otherwise. The student's t-test was used for statistical analysis of the data. $\mathrm{P}<0.05$ was considered to be statistically significant.

\section{Results}

Establishment of a monoclonal MKN28-HSulf-1 stable cell line. To investigate the role of HSulf-1 in gastric cancer, we first established a gastric cell line that was able to stably express HSulf-1. As previously reported, endogenous HSulf-1 was downregulated in a number of gastric cancer cell lines including MKN28 (22). We therefore transfected pcDNA3.1/ Myc-His(-)HSulf-1 or an empty vector plasmid, pcDNA3.1/ Myc-His(-), into this cell line. Following G418 selection the monoclonal MKN28-HSulf-1 cell line was established, which expressed a significantly higher amount of HSulf-1 mRNA and protein than the empty vector control ( $\mathrm{Ctrl})$ as verified by both quantitative real-time RT-PCR (Fig. 1A) and immunoblotting (Fig. 1B). The results shown in Fig. 1 are representative of at least three independent experiments with similar results.

HSulf-1 expression inhibits gastric cancer cell proliferation. To determine the effect of HSulf-1 expression on gastric cancer cell proliferation, a MTS assay and a clone formation assay were utilized. Compared with control cells, HSulf-1-expressing monoclonal MKN28 cells showed a significantly reduced growth rate starting from day 4 (Fig. 2A). HSulf-1-expressing MKN28 cells also formed many fewer colonies, as shown in the colony formation assay (Fig. 2B). These data indicated that HSulf-1 expression may significantly suppress the growth and proliferation of MKN28 gastric cancer cells, consistent with previous studies showing that HSulf-1 inhibits the growth of a number of tumors, including ovarian, breast, myeloma and hepatocellular carcinoma cells (15-21). Data shown in Fig. 2 are representative of three independent experiments with similar results.

HSulf-1 is capable of inhibiting the Hh signaling pathway. HSulf-1 has emerged as a negative regulator of Hh signaling through the disruption of $\mathrm{Hh}$ signaling transduction by desulfating HSPG (17-19). Therefore, we investigated the effect of HSulf-1 on Hh signaling. Hh pathway activation was evaluated by a Gli luciferase reporter assay and semi-quantitative RT-PCR for Hh target genes. 8xGli luciferase reporter activity was significantly elevated in MKN28 cells as compared to mutant Gli reporter (Mut Gli)-transfected cells, indicating that the Hh pathway is activated in MKN28 cells (Fig. 3A). Notably, the introduction of HSulf-1 significantly inhibited 8xGli reporter activity (Fig. 3A, HSulf-1 vs. Ctrl), suggesting that HSulf-1 downregulates $\mathrm{Hh}$ signaling. Furthermore, HSulf-1 significantly downregulated the expression of $\mathrm{Hh}$ pathway target genes, including GLI1, PTCH1, PTCH2, HHIP, C-MYC, CCND1, FOXF1, FOXM1 and BCL2 (Fig. 3B). Taken together, these results markedly support that the expres- 
A

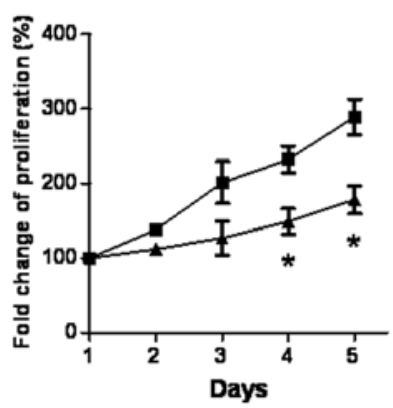

B

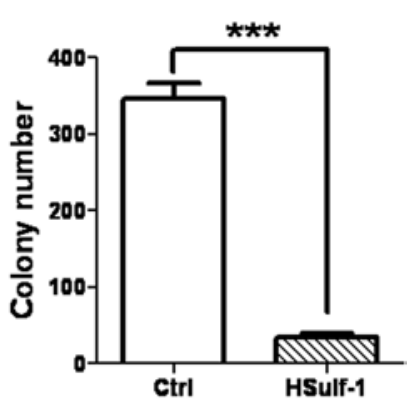

Figure 2. Effects of HSulf-1 on cell proliferation in gastric cancer cells. (A) Growth curves of MKN28-HSulf-1 (ム) and empty vector control (ロ) cells by MTS assay. Values are the mean \pm SD of four independent experiments. (B) Quantification of colony formation assay of MKN28-HSulf-1 and empty vector control cells performed in triplicate in three independent experiments. ${ }^{*} \mathrm{p}<0.05,{ }^{* * *} \mathrm{p}<0.001$ (Student's t-test). Error bars represent $\pm \mathrm{SD}$.

A

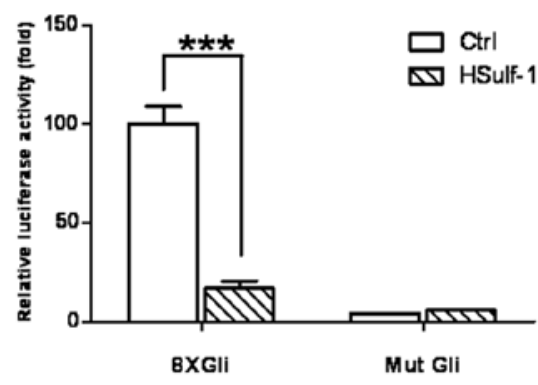

B

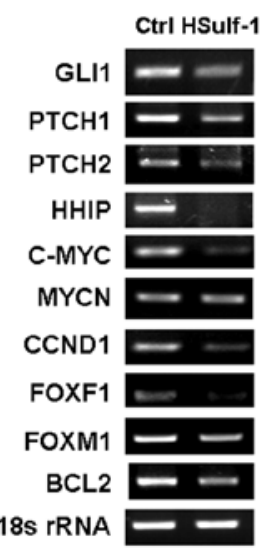

Figure 3. Effects of HSulf-1 on the Hh signaling pathway. (A) 8xGli luciferase reporter assay in HSulf-1 or empty vector (Ctrl)-transfected MKN28 cells. Mutant Gli reporter (Mut Gli) served as a control. *** $\mathrm{p}<0.001$ (Student's t-test). Error bars, \pm SD. (B) Semi-quantitative RT-PCR showing effects of HSulf- 1 on mRNA expression of $\mathrm{Hh}$ target genes. 18S rRNA served as a control. Data are representative of at least three independent experiments with similar results.

sion of HSulf-1 is capable of downregulating Hh signaling, correlating with its growth inhibition in gastric cancer cells. The data shown in Fig. 3 represent three independent experiments with similar results.

\section{Discussion}

In this study, HSulf-1 was found to play a significant role in suppressing the growth and proliferation of gastric cancer in MKN28 cells. Furthermore, it was determined that HSulf-1 expression in gastric cancer cells may significantly inhibit the Hh signaling pathway, providing new mechanistic insight into HSulf-1-mediated growth inhibition in gastric cancer.

Emerging evidence revealed that HSulf-1 may function as a novel tumor suppressor in various types of cancer (15-21). It was recently reported that HSulf-1 is downregulated in several types of tumors including ovarian, breast, hepatocellular carcinoma, renal tumor cells and head and neck squamous carcinoma cells (15-21). Re-expression of HSulf-1 in ovarian, hepatocellular carcinoma or head and neck squamous carcinoma cells retarded cell proliferation and motility, and enhanced stress-induced apoptosis (15-17,20). More significantly, HSulf-1 expression in breast, myeloma or hepatocelluar carcinoma cell-derived xenografts blocked angiogenesis and tumor invasion in vivo $(18,19)$. Notably, HSulf-1 is upregulated in primary pancreatic adenocarcinoma, and overexpression of
HSulf-1 reduced growth capacity but increased invasiveness in pancreatic cancer $(23,24)$. These studies suggested that HSulf-1 may play various roles in various types of cancer. Whether or not HSulf-1 plays a role in gastric cancer tumorigenicity remains to be elucidated. Our previous study suggested that HSulf-1 expression is downregulated in gastric cancer cells and that the gene silencing of HSulf-1 is associated with promoter hypermethylation (22). In the present study, we investigated whether HSulf-1 regulates the proliferation and growth of gastric cancer cells. HSulf-1 expression in MKN28 gastric cancer cells was found to markedly suppress cell proliferation and growth (Fig. 2A-B), consistent with previous studies, which revealed that HSulf-1 inhibits the proliferation of multiple types of cancer cells (15-21).

As a newly identified member of the endosulfatase family, HSulf-1 selectively desulfates cell surface HSPGs (12-14). Sulfated HSPGs play a pivotal role in mediating Wnt and Hh ligand distribution, stability and ligand-receptor binding $(12,14,25)$. Therefore, desulfation by HSulf- 1 re-expression may interfere with Wnt and Hh signaling $(14,25)$. Sulfated HSPGs also serve as co-receptors for multiple growth factors and cytokines. Thus, desulfation by HSulf-1 leads to the abrogation of several receptor tyrosine kinase signaling pathways, particularly heparin-binding growth factors including fibroblast growth factor 2, vascular endothelial growth factor, hepatocyte growth factor, PDGF and heparin-binding epidermal 
growth factor (15-18). Since aberrant Hh activation has been shown to play a role in the tumorigenesis of multiple cancers, including gastric cancer (6-8), and Hh signaling regulates cell growth and survival, we explored the hypothesis that HSulf-1 regulates the activity of $\mathrm{Hh}$ signaling in gastric cancer cells. Notably, the activated Gli transcription activity in MKN28 gastric cancer cells was eliminated by HSulf-1 expression (Fig. 3A), indicating that HSulf-1 may abrogate Hh signaling activity in gastric cancer. Furthermore, we examined the effect of HSulf-1 on the expression of Hh pathway target genes and observed the significant downregulation of GLI1, PTCH1, PTCH2, HHIP, C-MYC, CCND1, FOXF1, FOXM1 and BCL2 (Fig. 3B). As Hh target genes, GLI1, PTCH1/2 and HHIP are responsible for the fine-tuning of $\mathrm{Hh}$ signaling via positive and negative feedback loops $(10,11,26-28)$. The Hh pathway induces cell proliferation through upregulation of C-MYC, cell cycle regulator CCND1 and FOXM1, and promotes survival of cells via BCL2 (28-31). Collectively, the data confirmed that inhibition of $\mathrm{Hh}$ signaling may be one of the major mechanisms mediating HSulf-1-induced growth inhibition in gastric cancer cells. However, the results do not exclude the possibility that HSulf-1 may mediate its growth inhibition via multiple mechanisms including modulation of the Wnt and heparin-binding growth factor signaling pathways.

In conclusion, we demonstrated that HSulf-1 may function as a tumor suppressor in gastric cancer, as it significantly retarded cell growth and downregulated the $\mathrm{Hh}$ signaling pathway in gastric cancer cells. Since HSulf-1 potentiates the effects of histone deacetylase (HDAC) inhibitors (32), and its epigenetic silencing in ovarian cancer is implicated in chemoresistance (33), strategies targeting the epigenetic reactivation of HSulf-1 in combination with HDAC inhibitors may prove to be useful therapeutic modalities in treating gastric cancer (34).

\section{Acknowledgements}

This study was partially supported by funds from the National Key Basic Research Project (No. 2007CB914401), the National Key Basic Research and Development (973) Program of China (No. 06CB503905) and the China Natural Science Foundation (No. 30770475).

\section{References}

1. Hartgrink HH, Jansen EP, van Grieken NC and van de Velde CJ: Gastric cancer. Lancet 374: 477-490, 2009.

2. Ding SZ, Goldberg JB and Hatakeyama M: Helicobacter pylori infection, oncogenic pathways and epigenetic mechanisms in gastric carcinogenesis. Future Oncol 6: 851-862, 2010.

3. Merchant JL, Saqui-Salces M and El-Zaatari M: Hedgehog signaling in gastric physiology and cancer. Prog Mol Biol Trans Sci 96: 133-156, 2010

4. Martin J, Donnelly JM, Houghton J and Zavros Y: The role of sonic hedgehog reemergence during gastric cancer. Dig Dis Sci 55: 1516-1524, 2010.

5. Varjosalo $M$ and Taipale J: Hedgehog: functions and mechanisms. Genes Dev 22: 2454-2472, 2008.

6. Yang L, Xie G, Fan Q and Xie J: Activation of the hedgehog-signaling pathway in human cancer and the clinical implications. Oncogene 29: 469-481, 2010.

7. Scales SJ and de Sauvage FJ: Mechanisms of Hedgehog pathway activation in cancer and implications for therapy. Trends Pharmacol Sci 30: 303-312, 2009.

8. Ma X, Chen K, Huang S, et al: Frequent activation of the hedgehog pathway in advanced gastric adenocarcinomas. Carcinogenesis 26: 1698-1705, 2005.
9. Hooper JE and Scott MP: Communicating with Hedgehogs. Nat Rev Mol Cell Biol 6: 306-317, 2005.

10. Huangfu D and Anderson KV: Signaling from Smo to $\mathrm{Ci} /$ Gli: conservation and divergence of Hedgehog pathways from Drosophila to vertebrates. Development 133: 3-14, 2006.

11. Osterlund $\mathrm{T}$ and Kogerman P: Hedgehog signalling: how to get from Smo to Ci and Gli. Trends Cell Biol 16: 176-180, 2006.

12. Lin X: Functions of heparan sulfate proteoglycans in cell signaling during development. Development 131: 6009-6021, 2004.

13. Morimoto-Tomita M, Uchimura K, Werb Z, Hemmerich S and Rosen SD: Cloning and characterization of two extracellular heparin-degrading endosulfatases in mice and humans. J Biol Chem 277: 49175-49185, 2002.

14. Bernfield M, Gotte M, Park PW, et al: Functions of cell surface heparan sulfate proteoglycans. Annu Rev Biochem 68: 729-777, 1999.

15. Lai J, Chien J, Staub J, et al: Loss of HSulf-1 up-regulates heparin-binding growth factor signaling in cancer. J Biol Chem 278: 23107-23117, 2003.

16. Lai JP, Chien JR, Moser DR, et al: hSulf1 Sulfatase promotes apoptosis of hepatocellular cancer cells by decreasing heparin-binding growth factor signaling. Gastroenterology 126 : 231-248, 2004.

17. Lai JP, Sandhu DS, Shire AM and Roberts LR: The tumor suppressor function of human sulfatase 1 (SULF1) in carcinogenesis. J Gastrointest Cancer 39: 149-158, 2008.

18. Narita K, Staub J, Chien J, et al: HSulf-1 inhibits angiogenesis and tumorigenesis in vivo. Cancer Res 66: 6025-6032, 2006.

19. Dai Y, Yang Y, MacLeod V, et al: HSulf-1 and HSulf-2 are potent inhibitors of myeloma tumor growth in vivo. J Biol Chem 280: 40066-40073, 2005.

20. Lai JP, Chien J, Strome SE, et al: HSulf-1 modulates HGF-mediated tumor cell invasion and signaling in head and neck squamous carcinoma. Oncogene 23: 1439-1447, 2004.

21. Lai JP, Sandhu DS, Moser CD, et al: Additive effect of apicidin and doxorubicin in sulfatase 1 expressing hepatocellular carcinoma in vitro and in vivo. J Hepatol 50: 1112-1121, 2009.

22. Chen Z, Fan JQ, Li J, et al: Promoter hypermethylation correlates with the Hsulf-1 silencing in human breast and gastric cancer. Int J Cancer 124: 739-744, 2009.

23. Li J, Kleeff J, Abiatari I, et al: Enhanced levels of Hsulf-1 interfere with heparin-binding growth factor signaling in pancreatic cancer. Mol Cancer 4: 14, 2005.

24. Abiatari I, Kleeff J, Li J, Felix K, Buchler MW and Friess H: Hsulf-1 regulates growth and invasion of pancreatic cancer cells. J Clin Pathol 59: 1052-1058, 2006.

25. Dhoot GK, Gustafsson MK, Ai X, Sun W, Standiford DM and Emerson CP, Jr.: Regulation of Wnt signaling and embryo patterning by an extracellular sulfatase. Science 293: 1663-1666, 2001.

26. Chuang PT, Kawcak T and McMahon AP: Feedback control of mammalian Hedgehog signaling by the Hedgehog-binding protein, Hip1, modulates Fgf signaling during branching morphogenesis of the lung. Genes Dev 17: 342-347, 2003.

27. Chuang PT and McMahon AP: Vertebrate Hedgehog signalling modulated by induction of a Hedgehog-binding protein. Nature 397: 617-621, 1999.

28. Katoh Y and Katoh M: Hedgehog target genes: mechanisms of carcinogenesis induced by aberrant hedgehog signaling activation. Curr Mol Med 9: 873-886, 2009.

29. Rao G, Pedone CA, Coffin CM, Holland EC and Fults DW: c-Myc enhances sonic hedgehog-induced medulloblastoma formation from nestin-expressing neural progenitors in mice. Neoplasia 5: 198-204, 2003.

30. Sherr CJ and Roberts JM: CDK inhibitors: positive and negative regulators of G1-phase progression. Genes Dev 13: 1501-1512, 1999.

31. Bigelow RL, Chari NS, Unden AB, et al: Transcriptional regulation of bcl-2 mediated by the sonic hedgehog signaling pathway through gli-1. J Biol Chem 279: 1197-1205, 2004.

32. Lai JP, Yu C, Moser CD, et al: SULF1 inhibits tumor growth and potentiates the effects of histone deacetylase inhibitors in hepatocellular carcinoma. Gastroenterology 130: 2130-2144, 2006.

33. Staub J, Chien J, Pan Y, et al: Epigenetic silencing of HSulf-1 in ovarian cancer:implications in chemoresistance. Oncogene 26: 4969-4978, 2007.

34. Lai JP, Thompson JR, Sandhu DS and Roberts LR: Heparin-degrading sulfatases in hepatocellular carcinoma: roles in pathogenesis and therapy targets. Future Oncol 4: 803-814, 2008. 